\title{
An Effective Segmentation Method for Iris Recognition System
}

\author{
Richard Yew Fatt Ng, Yong Haur Tay and Kai Ming Mok \\ Computer Vision and Intelligent Systems (CVIS) Group \\ Universiti Tunku Abdul Rahman, Malaysia \\ richng01@yahoo.com, \{tayyh, mokkm\}@mail.utar.edu.my
}

Keywords: iris recognition, iris segmentation, feature extraction, template matching, noise removal

\begin{abstract}
Iris recognition has become a popular research in recent years due to its reliability and nearly perfect recognition rates. Iris recognition system has three main stages: image preprocessing, feature extraction and template matching. In the preprocessing stage, iris segmentation is critical to the success of subsequent feature extraction and template matching stages. If the iris region is not correctly segmented, the eyelids, eyelashes, reflection and pupil noises would present in the normalized iris region. The presence of noises will directly deteriorate the iris recognition accuracy. The proposed approach gives a solution for compensating all four types of noises to achieve higher accuracy rate. It consists of four parts: (a) Pupil is localized using thresholding and Circular Hough Transform methods. (b) Two search regions including the outer iris boundaries are defined to locate the outer iris. (c) Two search regions are selected based on pupil position to detect the upper and lower eyelids. (d) Thresholding is implemented to remove eyelashes, reflection and pupil noises. The method evaluates on iris images taken from the CASIA iris image database version 1.0 [1] Experimental results show that the proposed approach has achieved high accuracy of $98.62 \%$.
\end{abstract}

\section{Introduction}

Biometric identification is an emerging technology which gains more attention in recent years. It employs physiological or behavioural characteristics to identify an individual. The physiological characteristics are iris, fingerprint, face and hand geometry. Voice, signature and keystroke dynamics are classified as behavioural characteristics. Among these characteristics, iris has distinct phase information which spans about 249 degrees of freedom [6,7]. This advantage let iris recognition be the most accurate and reliable biometric identification.

The three main stages of an iris recognition system are image preprocessing, feature extraction and template matching.

The iris image needs to be preprocessed to obtain useful iris region. Image preprocessing is divided into three steps: iris localization, iris normalization and image enhancement. Iris localization detects the inner and outer boundaries of iris.
Eyelids and eyelashes that may cover the iris region are detected and removed. Iris normalization converts iris image from Cartesian coordinates to Polar coordinates. The iris image has low contrast and non-uniform illumination caused by the position of the light source. All these factors can be compensated by the image enhancement algorithms.

Feature extraction uses texture analysis method to extract features from the normalized iris image. The significant features of the iris are extracted for accurate identification purpose.

Template matching compares the user template with templates from the database using a matching metric. The matching metric will give a measure of similarity between two iris templates. It gives a range of values when comparing templates from the same iris, and another range of values when comparing templates from different irises. Finally, a decision with high confidence level is made to identify whether the user is an authentic or imposter.

Iris segmentation localizes the correct iris region in an eye image. It is critical to the success of subsequent feature extraction and template matching stages. This paper proposes a solution for compensating all four types of noises to achieve higher accuracy rate.

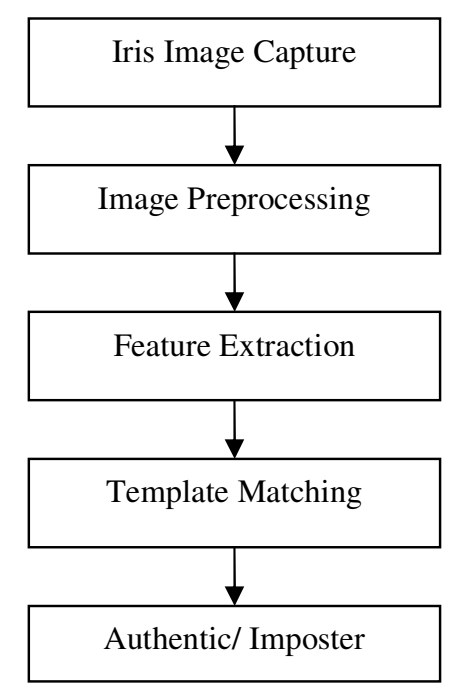

Figure 1: Stages of iris recognition algorithm. 
Related work is introduced in the next section. Proposed iris segmentation method is presented in Section 3. Section 4 discusses iris normalization and image enhancement algorithms. Section 5 and Section 6 describe details of feature extraction and template matching stages. Experimental results are illustrated in Section 7 while the conclusion is drawn in the last section.

\section{Related work}

Performance of the iris segmentation method will directly affect the recognition accuracy. Different iris segmentation methods have been proposed for improving the recognition accuracy.

Daugman [6,7] proposed an Integro-differential operator for locating the inner and outer boundaries of iris, as well as the upper and lower eyelids. The operator computes the partial derivative of the average intensity of circle points, with respect to increasing radius, $r$. After convolving the operator with Gaussian kernel, the maximum difference between inner and outer circle will define the center and radius of the iris boundaries. For upper and lower eyelids detection, the path of contour integration is modified from circular to parabolic curve.

Wildes [9] used edge detection and Hough transform to localize the iris. Edge detector is applied to a gray scale iris image to generate the edge map. Gaussian filter is applied to smooth the image to select the proper scale of edge analysis. The voting procedure is realized using Hough transform in order to search for the desired contour from the edge map. The center coordinate and radius of the circle with maximum number of edge points is defined as the contour of interest. For eyelids detection, the contour is defined using parabolic curve parameter instead of the circle parameter.

Black hole search method is proposed by C. Teo and H. Ewe [2] to compute the center and area of a pupil. Since the pupil is the darkest region in the image, this approach applies threshold segmentation method to find the dark areas in the iris image. The dark areas are called as "black holes". The center of mass of these black holes is computed from the global image. The area of pupil is the total number of those black holes within the region. The radius of the pupil can be calculated from the circle area formula.

Cui et al. [5] decomposed the iris image using Haar wavelet before pupil localization. Modified Hough Transform was used to obtain the center and radius of pupil. Iris outer boundary was localized using an integral differential operator. Texture segmentation is adopted to detect upper and lower eyelids. The energy of high spectrum at each region is computed to segment the eyelashes. The region with high frequency is considered as the eyelashes area. The upper eyelashes are fit with a parabolic arc. The parabolic arc shows the position of the upper eyelid. For lower eyelid detection, the histogram of the original image is used. The lower eyelid area is segmented to compute the edge points of the lower eyelid. The lower eyelid is fit with the edge points.

W. Kong et al. [10] proposed Gabor filter and variance of intensity approaches for eyelash detection. The eyelashes are categorized into separable eyelashes and multiple eyelashes.
Separable eyelashes are detected using 1D Gabor filters. A low output value is obtained from the convolution of the separable eyelashes with the Gabor filter. For multiple eyelashes, the variance of intensity is very small. If the variance of intensity in a window is smaller than a threshold, the center of the window is considered as the eyelashes.

\section{Iris segmentation}

This section discusses in detail the proposed iris segmentation method. It includes iris inner and outer boundaries localization, upper and lower eyelids detection and eyelashes, reflection and pupil noises removal algorithms.

\subsection{Iris inner boundary localization}

As pupil is a black circular region, it is easy to detect the pupil inside an eye image [4,8,11]. Firstly, pupil is detected using thresholding operation. An appropriate threshold is selected to generate the binary image which contains pupil only. Morphological operator is applied to the binary image to remove the reflection inside the pupil region and other dark spots caused by eyelashes. Figure 2(b) shows the binary image after thresholding and morphological operator.

Since the inner boundary of an iris can be approximately modelled as circles, circular Hough transform is used to localize the iris $[9,10]$. Firstly, edge detector is applied to binary image to generate the edge map. The edge map is obtained by calculating the first derivative of intensity values and thresholding the results. The formula is defined as

$$
g(x, y)=\left|\nabla G_{\sigma}(x, y) * I(x, y)\right|
$$

where $\nabla \equiv\left(\frac{\partial}{\partial x}, \frac{\partial}{\partial y}\right)$ and

$$
\mathrm{G}_{\sigma}(\mathrm{x}, \mathrm{y})=\frac{1}{2 \pi \sigma^{2}} \mathrm{e}^{-\frac{\left(\mathrm{x}-\mathrm{x}_{0}\right)^{2}+\left(y-y_{0}\right)^{2}}{2 \sigma^{2}}}
$$

denotes a two dimension Gaussian filter of scale $\sigma$. Gaussian filter is applied to smooth the image to select the proper scale of edge analysis. It filters the random edges which are irrelevant to reduce false circle detection.

The voting procedure is realized using circular Hough Transform in order to search for the desired contour from the edge map. Assuming a circle with centre coordinate $\left(x_{c}, y_{c}\right)$ and radius $r$, each edge point on the circle casts a vote in Hough space. The circular contour of interest is defined as

$$
\left(x_{i}-x_{c}\right)^{2}+\left(y_{i}-y_{c}\right)^{2}=r^{2}
$$

The centre coordinate and radius of the circle with maximum number of votes are defined as the pupil centre and iris inner boundary respectively. If the number of votes is less than a certain threshold set by circular Hough Transform, it is assumed that eye is not presented in the image, it is heavily 
occluded by eyelids, it is in defocused or motion blurred condition.

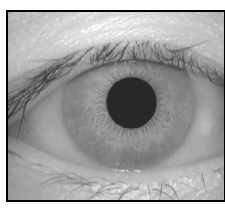

(a)

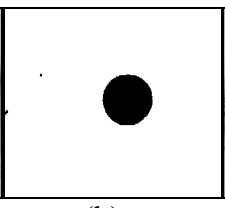

(b)

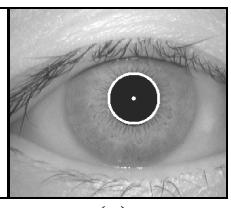

(c)
Figure 2:(a) Original eye image. (b) Binary image after thresholding and morphological operator. (c) Pupil localization.

\subsection{Iris outer boundary localization}

In order to locate the iris outer boundary, the proposed method selects two search regions including the outer iris boundaries. To reduce computational time, localization is limited to the search regions only. The right search region and left search region are shown in Figure 3(a). The pupil centre is referred as origin. The search region is a sector with radius from pupil boundary to a maximum radius. Maximum radius is defined as the distance from pupil centre to boundaries of the right or left search region.

$$
\begin{aligned}
& r_{\text {right }}=\min \left(\text { width }-x_{c}, \text { max_threshold }\right) \\
& r_{\text {left }}=\min \left(x_{c}, \max \_t h r e s h o l d\right)
\end{aligned}
$$

where $r_{\text {right }}$ denotes the maximum radius of the right search region and $r_{\text {left }}$ denotes the maximum radius of the left search region. Maximum threshold is a constant defined based on the iris size. The minimum radius of the search regions starts ten pixels away from the pupil boundary. This is to avoid the effect caused by the pupil noise. In order to avoid occlusion caused by eyelashes, upper and lower eyelids, the search regions are selected on the lower iris region.

The intensities of each radius in the two search regions are added up according to Equation (6). The sum of intensities of each radius is calculated to reduce the effects caused by noises and variations of iris texture. The negative sign in the Equation (6) indicates that the y-coordinate starts from top to bottom of the image.

$$
I[r, \theta]=\left(y_{c}-r \sin \theta\right) * \text { width }+\left(x_{c}+r \cos \theta\right)
$$

Finally, the iris outer boundary, $R_{\text {iris }}$ can be calculated using Equation (9). The right and left iris boundaries are the maximum difference between the sum of intensities of two outer radius and two inner radius. The iris outer boundary is the average of the distances from pupil centre to right iris boundary, $R_{r}$ and left iris boundary, $R_{l}$.

$$
\begin{gathered}
R_{r}=\arg \max _{r}\{I[r+2]+I[r+1]-I[r-1]-I[r-2]\} \\
R_{l}=\arg \max _{r}\{I[r+2]+I[r+1]-I[r-1]-I[r-2]\} \\
R_{\text {iris }}=\frac{R_{r}+R_{l}}{2}
\end{gathered}
$$

The iris centre $\left(x_{i r i s}, y_{\text {iris }}\right)$ is defined in Equation (10) and (11). The $\mathrm{x}$-coordinate of the iris centre shifts from the pupil centre depending on the difference between $R_{r}$ and $R_{l}$. The ycoordinate of the iris centre is same with the pupil centre.

$$
\begin{gathered}
x_{\text {iris }}=x_{c}+\frac{\left(R_{r}-R_{l}\right)}{2} \\
y_{\text {iris }}=y_{c}
\end{gathered}
$$

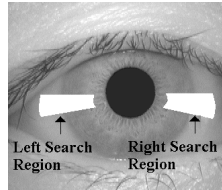

(a)

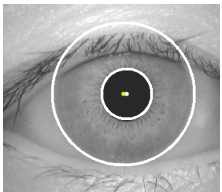

(b)
Figure 3:(a) Right and left search regions of the iris image. (b) Iris inner and outer boundaries localization.

\subsection{Upper and lower eyelids detection}

Similar to iris outer boundary localization, the proposed method selects two search regions to detect upper and lower eyelids. The upper and lower search regions are labelled as in Figure 4(a). The pupil centre, iris inner and outer boundaries are used as reference to select the two search regions.

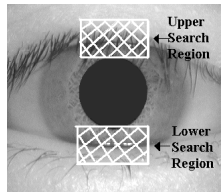

(a)

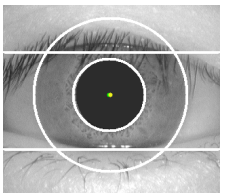

(b)
Figure 4:(a) Upper and lower search regions of the iris image. (b) Upper and lower eyelids detection.

The search regions are confined within the inner and outer boundaries of the iris. The width of the two search regions is same with diameter of the pupil.

Sobel edge detection is applied to the search regions to detect the eyelids. In order to reduce the false edges detection caused by eyelashes, Sobel kernel is tuned to the horizontal direction.

\begin{tabular}{|l|l|l|}
\hline-1 & -2 & -1 \\
\hline 0 & 0 & 0 \\
\hline 1 & 2 & 1 \\
\hline
\end{tabular}

Table 1: Sobel kernel tuned to horizontal direction.

After edge detection step, the edge image is generated. The eyelids are detected using linear Hough Transform method. The method calculates total number of edge points in every horizontal row inside the search regions. The horizontal row with maximum number of edge points is selected as eyelid boundary. If the maximum number of edge points is less than a predefined threshold, it is assumed that eyelid is not presented in the search regions. The eyelids detection process is illustrated in Figure 5. 
In the proposed method, the eyelid boundaries are approximately modelled as straight lines. Edge detection cannot identify all pixels along the eyelid boundaries. The eyelid boundaries are normally occluded by the eyelashes. Therefore, eyelid boundaries are modelled with straight lines approximation.

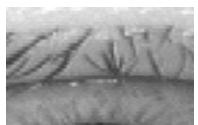

(a)

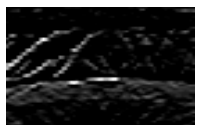

(b)

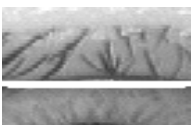

(c)
Figure 5:(a) Upper search region of the iris image. (b) Upper search region after Sobel edge detection. (c) Upper eyelid detection.

\subsection{Eyelashes, reflection and pupil noise removal}

There are two types of eyelashes grown at the edge of the eyelids, separable eyelash and multiple eyelashes [10]. Separable eyelash can be distinguished from other eyelashes. Multiple eyelashes are several eyelashes overlap in a small region. Eyelashes appear randomly inside the iris region. It is difficult to detect the eyelashes effectively. The eyelashes are observed to have low intensity values. A simple thresholding technique is applied to segment eyelashes accurately.

In general, iris imaging device uses near infrared light as illumination source. Near Infrared (NIR) illuminator is used to reveal complex textures for darkly pigmented irises. Reflection regions are characterized by high intensity values close to 255. A high threshold value can be used to separate the reflection noise.

The pupil area is not necessary a circular region. When the pupil boundary is approximately modelled as circle, some parts of pupil will exist inside normalized iris region as noise. Similar to eyelashes and reflection detection, a threshold is applied to remove the pupil noise.

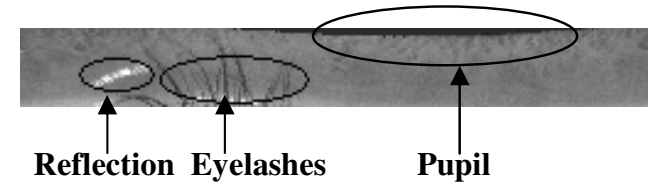

Figure 6: Normalized iris image with pupil, eyelashes and reflection noises.

\section{Normalization and enhancement}

Iris may be captured in different size with varying imaging distance. Due to illumination variations, the radial size of the pupil may change accordingly. Therefore, the iris region needs to be normalized to compensate for these variations. Figure 6 shows the iris image after normalization.

Normalization remaps each pixel in the localized iris region from the Cartesian coordinates to polar coordinates. The nonconcentric polar representation is normalized to a fixed size rectangular block.
The normalized iris image has low contrast and non-uniform illumination caused by the light source position. The image needs to be enhanced to compensate for these factors.

Local histogram analysis is applied to the normalized iris image to reduce the effect of non-uniform illumination and obtain well-distributed texture image.

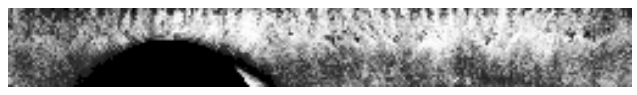

Figure 7: Enhanced iris image.

\section{Feature extraction}

1D Log Gabor filter is used to extract iris features from the normalized iris image. A Log Gabor filter is a Gaussian transfer function on a logarithmic scale [3]. It has strictly band pass filter to remove the DC components caused by background brightness.

$G(w)=\exp \left(\left(-\log \left(w / w_{0}\right)^{2}\right) / 2\left(\log \left(k / w_{0}\right)\right)^{2}\right)$

where $w_{0}$ denotes the filter's centre frequency and $k$ denotes the bandwidth of the filter.

Each pattern is demodulated to extract its phase information. The phase information is quantized into four quadrants in the complex plane. Each quadrant is represented with two bits phase information. Therefore, each pixel in the normalized image is demodulated into two bits code in the template.

The phase information is extracted because it provides the significant information within the iris region. It does not depend on extraneous factors, such as imaging contrast, illumination and camera gain.

\section{Template matching}

Hamming distance is defined as the fractional measure of dissimilarity between two binary templates [6,7]. A value of zero would represent a perfect match. The two templates that are completely independent would give a Hamming distance near to 0.5 . A threshold is set to decide the two templates are from the same person or different persons.

The fractional hamming distance is sum of the exclusive-OR between two templates over the total number of bits. Masking templates are used in the calculation to exclude the noise regions. Only those bits in the templates that correspond to ' 1 ' bit in the masking template will be used in the calculation.

$$
H D=\frac{\|(\text { template } A \otimes \text { template } B) \bigcap \text { mask } A \cap \text { mask } B \|}{\| \text { mask } A \cap \operatorname{maskB} \|}
$$

\section{Experimental results}

The proposed algorithm was evaluated using CASIA iris image database version 1.0 [1]. There are 756 iris images from 108 different irises. For each eye, 7 images are captured in two sessions. The time interval between two sessions is 
about one month. The resolution of the iris images is $320 \times 280$ pixels.

\begin{tabular}{|l|l|l|}
\hline Method & Iris inner boundary & Iris outer boundary \\
\hline Cui et al. [6] & $99.34 \%$ & $99.34 \%$ \\
\hline Proposed & $99.07 \%$ & $98.68 \%$ \\
\hline
\end{tabular}

Table 2: Comparison of iris inner and outer boundaries detection rate with other algorithm.

\begin{tabular}{|l|l|l|}
\hline Method & Upper eyelid & Lower eyelid \\
\hline Cui et al. [6] & $97.35 \%$ & $93.39 \%$ \\
\hline Proposed & $95.77 \%$ & $95.37 \%$ \\
\hline
\end{tabular}

Table 3: Comparison of upper and lower eyelids detection rate with other algorithm.

In Table 2 and Table 3, the detection rates are observed by eyes because there is no standard method for evaluating the localization results. Since the iris segmentation results on CASIA iris image database version 1.0 is shown in [5], the performance of our proposed method is compared with their method in Table 2 and Table 3. It shows that our proposed method is comparable with their method.

The detection rates of the iris inner and outer boundary are $99.07 \%$ and $98.68 \%$ respectively. The false localization of iris inner boundary is caused by the pupil that is not a perfect circle. The algorithms try to find the best circle which fits the pupil boundary. Iris outer boundary is detected incorrectly due to the presence of eyelashes and the iris outer boundary is too near to the image boundary.

The accuracy of upper and lower eyelids detection are $95.77 \%$ and $95.37 \%$ as shown in Table 3 . The detection rate of the eyelid boundaries is significantly less than detection rate of iris boundaries. The eyelid boundaries are usually covered by eyelashes. Furthermore, it is difficult to model the eyelid boundaries in parabolic shape. The presence of skin fold also causes false eyelids detection.

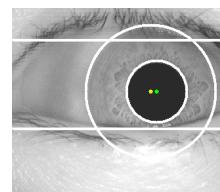

(a)

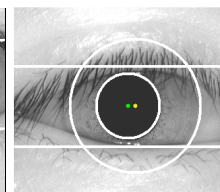

(b)

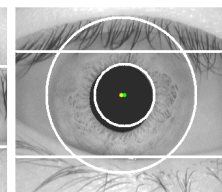

(c)
Figure 8: Inaccurate segmentation due to (a) iris outer boundary near to image boundary. (b) presence of eyelashes. (c) pupil is not a perfect circle.

\section{ROC Curve}

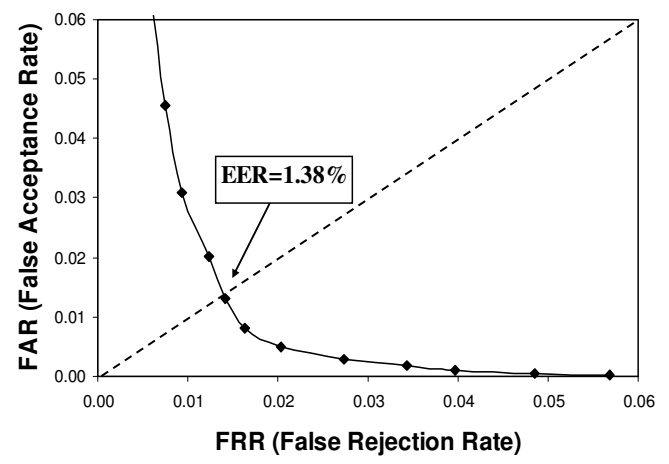

Figure 9: ROC curve for iris recognition results.

ROC curve is plotted to measure the recognition accuracy. From the experimental results, the algorithm shows an overall accuracy of $98.62 \%$ with Equal Error Rate (EER) of $1.38 \%$. It is noted that the result is not perfect due to the low quality of the iris images. The iris region is heavily occluded by eyelids and eyelashes or distorted much due to pupil dilation and constriction. Some of the iris images are in defocused or motion blurred condition as shown in Figure 10. Image quality assessment is needed to select clear images with high quality.

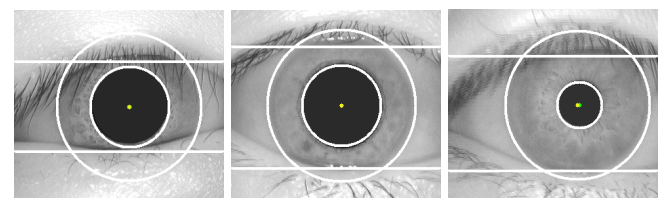

(a)

(b)

(c)

Figure 10: (a) An occluded eye. (b) A defocused eye. (c) A motion blurred eye.

\section{Conclusions}

The accuracy of iris recognition is dependent on the performance of the iris segmentation method. An effective iris segmentation method for iris recognition system is presented in this paper. It proposes a solution for compensating all types of noises to achieve higher accuracy rate. Four types of noises exist in a normalized iris region are eyelids, eyelashes, pupil and reflection. Circular Hough Transform is used to locate the iris inner boundary. The method makes use of search regions to locate iris outer boundary and eyelids. Thresholding operation is applied to remove the pupil, reflection and eyelashes noises. The experimental results show that the proposed iris segmentation method is effective. The approach has achieved a high recognition rate up to $98.62 \%$.

\section{Acknowledgements}

The author would like to acknowledge Institute of Automation, Chinese Academy of Science for providing 
CASIA iris image database [1]. This research is partially funded by Malaysia MOSTI ScienceFund 01-02-11-SF0019.

\section{References}

[1] "CASIA Iris Image Database,"

http://www.sinobiometrics.com/Databases.htm, (2007).

[2] C.C. Teo and H.T. Ewe (2005). "An Efficient OneDimensional Fractal Analysis for Iris Recognition", Proceedings of the $13^{\text {th }}$ WSCG International Conference in Central Europe on Computer Graphics, Visualization and Computer Vision 2005, pp. 157-160.

[3] D. Field. "Relations between the statistics of natural images and the response properties of cortical cells", Journal of the Optical Society of America, (1987).

[4] G. Xu, Z. Zhang and Y. Ma. "Automatic Iris Segmentation Based on Local Areas", The $18^{\text {th }}$ International Conference on Pattern Recognition, (2006).

[5] J. Cui, Y. Wang, T. Tan. L. Ma and Z. Sun. "A Fast and Robust Iris Localization Method Based on Texture Segmentation", Proceedings of the SPIE, vol. 5404, pp. 401408, (2004).

[6] J. Daugman. "High Confidence Visual Recognition of Persons by a Test of Statistical Independence", IEEE Tans. Pattern Analysis and Machine Intelligence, vol.15, pp.11481161, (1993).

[7] J. Daugman. "How Iris Recognition Works", IEEE Trans. CSVT, vol. 14, no. 1, pp. $21-30,(2004)$.

[8] K. Grabowski, W. Sankowski, M. Zubert, and M. Napieralska. "Reliable Iris Localization Method with Application to Iris Recognition in Near Infrared Light", MIXDES 2006, (2006).

[9] R.P. Wildes. "Iris Recognition: An Emerging Biometric Technology", Proceedings of the IEEE, vol.85, pp.13481363, (1997).

[10] W. Kong and D. Zhang. "Accurate iris segmentation based on novel reflection and eyelash detection model", Proceedings of 2001 International Symposium on Intelligent Multimedia, Video and Speech Processing, (2001).

[11] W. Sankowski, K. Grabowski, and M. Napieralska M. Zubert. "Eyelids Localization Method Designed for Iris Recognition System”,_MIXDES 2007, (2007). 